\title{
Testing quantum correlations versus single-particle properties within Leggett's model and beyond
}

\author{
CYRIL BRANCIARD ${ }^{*}$, NICOLAS BRUNNER ${ }^{1}$, NICOLAS GISIN ${ }^{1}$, CHRISTIAN KURTSIEFER ${ }^{2}$, \\ ANTIA LAMAS-LINARES², ALEXANDER LING² AND VALERIO SCARANI ${ }^{2}$ \\ ${ }^{1}$ Group of Applied Physics, University of Geneva, 20 Rue de l'Ecole de Médecine, CH-1211 Geneva 4, Switzerland \\ ${ }^{2}$ Centre for Quantum Technologies/Physics Department, National University of Singapore, 117543 Singapore \\ *e-mail: cyril.branciard@physics.unige.ch
}

Quantum theory predicts and experiments confirm that nature can produce correlations between distant events that are nonlocal in the sense of violating a Bell inequality ${ }^{1}$. Nevertheless, Bell's strong sentence 'Correlations cry out for explanations' (ref. 2) remains relevant. The maturing of quantum information science and the discovery of the power of non-local correlations, for example for cryptographic key distribution beyond the standard quantum key distribution schemes ${ }^{3-5}$, strengthen Bell's wish and make it even more timely. In 2003, Leggett proposed an alternative model for non-local correlations ${ }^{6}$ that he proved to be incompatible with quantum predictions. We present here a new approach to this model, along with new inequalities for testing it. These inequalities can be derived in a very simple way, assuming only the non-negativity of probability distributions; they are also stronger than previously published and experimentally tested Leggett-type inequalities ${ }^{6-9}$. The simplest of the new inequalities is experimentally violated. Then we go beyond Leggett's model, and show that we cannot ascribe even partially defined individual properties to the components of a maximally entangled pair.

Formally, a correlation is a conditional probability distribution $P(\alpha, \beta \mid \mathbf{a}, \mathbf{b})$, where $\alpha, \beta$ are the outcomes observed by two partners, Alice and Bob, when they make measurements labelled by $\mathbf{a}$ and $\mathbf{b}$, respectively. On the abstract level, $\mathbf{a}$ and $\mathbf{b}$ are merely inputs, freely and independently chosen by Alice and Bob. On a more physical level, Alice and Bob hold two subsystems of a quantum state; in the simple case of qubits, the inputs are naturally characterized by vectors on the Poincaré sphere, hence the notation $\mathbf{a}, \mathbf{b}$.

How should we understand non-local correlations, in particular those corresponding to entangled quantum states? A natural approach consists in decomposing $P(\alpha, \beta \mid \mathbf{a}, \mathbf{b})$ into a statistical mixture of hopefully simpler correlations:

$$
P(\alpha, \beta \mid \mathbf{a}, \mathbf{b})=\int \mathrm{d} \lambda \rho(\lambda) P_{\lambda}(\alpha, \beta \mid \mathbf{a}, \mathbf{b}) .
$$

Bell's locality assumption is $P_{\lambda}(\alpha, \beta \mid \mathbf{a}, \mathbf{b})=P_{\lambda}^{\mathrm{A}}(\alpha \mid \mathbf{a}) P_{\lambda}^{\mathrm{B}}(\beta \mid \mathbf{b})$, admittedly the simplest choice, but an inadequate one as it turns out: quantum correlations violate Bell's locality ${ }^{1}$. Setting out to explore other choices, it is natural to require first that the $P_{\lambda}$ fulfil the so-called no-signalling condition, that is, that none of the correlations $P_{\lambda}$ results from a communication between Alice and Bob. This can be guaranteed by ensuring spacelike separation between Alice and Bob. Non-signalling correlations happen without any time ordering: there is not a first event, let us say on Alice's side, that causes the second event via some spooky action at a distance. We may phrase it differently: nonsignalling correlations happen from outside space-time, in the sense that there is no story in space-time that tells us how they happen. This is the case in orthodox quantum physics, or in some illuminating toy models such as the non-local box of Popescu and Rohrlich (PR box $)^{10}$. Mathematically, the no-signalling condition reads $P_{\lambda}(\alpha \mid \mathbf{a}, \mathbf{b})=P_{\lambda}(\alpha \mid \mathbf{a})$ and $P_{\lambda}(\beta \mid \mathbf{a}, \mathbf{b})=P_{\lambda}(\beta \mid \mathbf{b})$ : Alice's local statistics are not influenced by Bob's choice of measurement, and reciprocally.

In 2003, Leggett proposed another model of the form (1), which can also be experimentally tested against quantum predictions ${ }^{6}$. This model was recently brought into focus by the work of Gröblacher et al. ${ }^{7}$. The basic assumption of Leggett's model is that locally everything happens as if each single quantum system would always be in a pure state. We shall be concerned here with the case of binary outcomes $\alpha, \beta= \pm 1$, though generalizations are possible. In this case, the supplementary variables $\lambda$ in Leggett's model describe pure product states of two qubits, denoted by normalized vectors $\mathbf{u}, \mathbf{v}$ on the Poincaré sphere,

$$
\lambda=|\mathbf{u}\rangle \otimes|\mathbf{v}\rangle,
$$

and the local expectation values have the usual form as predicted by quantum physics:

$$
\begin{aligned}
& \langle\alpha\rangle_{\lambda}=\langle\mathbf{u}|\mathbf{a} \cdot \boldsymbol{\sigma}| \mathbf{u}\rangle=\mathbf{u} \cdot \mathbf{a}, \\
& \langle\beta\rangle_{\lambda}=\langle\mathbf{v}|\mathbf{b} \cdot \boldsymbol{\sigma}| \mathbf{v}\rangle=\mathbf{v} \cdot \mathbf{b} .
\end{aligned}
$$

If the qubits are encoded in the polarization of photons, as in Leggett's initial idea, then the assumption is that each photon should locally behave as if it were perfectly polarized (in the directions $\mathbf{u}$ and $\mathbf{v}$ ), and the local observations, conditioned on each $\lambda$, should fulfil Malus's law. It is worth emphasizing that Leggett's assumption concerns exclusively the local marginals $\langle\alpha\rangle_{\lambda}$ and $\langle\beta\rangle_{\lambda}$ of the probability distributions $P_{\lambda}$, whereas nothing is specified 


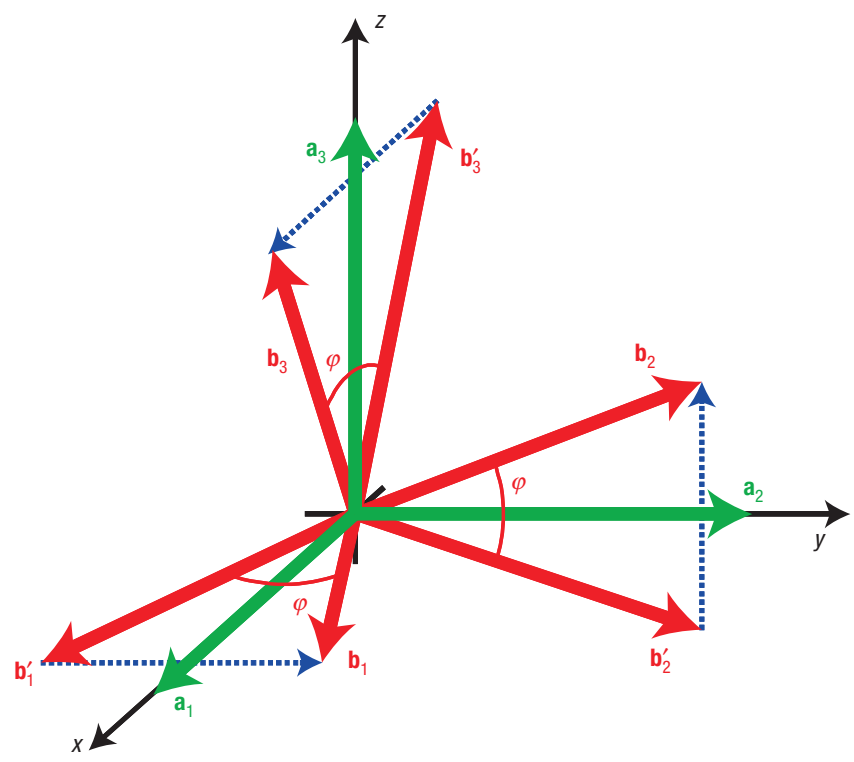

Figure 1 Alice's (green) and Bob's (red) measurement settings used to test inequality (4). In order for inequality (4) to hold, Bob's settings must be such that the three pairs $\left(\mathbf{b}_{i}, \mathbf{b}_{i}^{\prime}\right)$ form the same angle $\varphi$, and the three directions $\mathbf{e}_{i}$ of $\mathbf{b}_{i}-\mathbf{b}_{i}^{\prime}$ (blue) are orthogonal. The largest violation is obtained when Alice's settings $\mathbf{a}_{i}$ are chosen to be along the directions of $\mathbf{b}_{i}+\mathbf{b}_{i}^{\prime}$.

about the correlation coefficients $\langle\alpha \beta\rangle_{\lambda}$. Leggett's model can thus still be non-local, and can in general violate a Bell inequality.

Both in the original paper ${ }^{6}$ and in ref. 7, the model was presented by implicitly assuming a time ordering of the events (indeed, Bob's output is assumed to depend on Alice's input: her input must therefore have been defined before Bob's output materializes). Any model based on such an assumption had already been falsified by the so-called before-before experiment ${ }^{11,12}$, as Suarez emphatically stressed ${ }^{13}$. However, assumptions (2)-(3) clearly define non-signalling correlations, and Leggett's model can be defined without any reference to time ordering. As a consequence, its study does add something to our understanding of quantum non-locality. But what exactly? In what are such $P_{\lambda}$ 'simpler' than the usual quantum correlations? To answer these questions, we recall that, in quantum theory, the singlet state is such that the properties of the pair are sharply defined (the state is pure), but the properties of the individual particles are not. In this perspective, Leggett's model is an attempt at keeping the correlations and reintroducing sharp properties at the individual level as well.

Leggett's model cannot reproduce the correlations of the singlet state. Experimental falsifications have already been reported, first under additional assumptions ${ }^{7}$, then more directly $y^{8,9}$. These works relied on the violation of so-called Leggett-type inequalities. Analogous to Bell's inequalities, these criteria say that, under Leggett's assumptions (2) and (3), a measurable quantity $L$ should satisfy $L \leq L_{\max }$, whereas quantum theory predicts that $L>L_{\max }$ can be observed for suitable measurements. An important feature of Leggett-type inequalities is that, in contrast to Bell's inequalities, the bound $L_{\max }$ is not a fixed number: instead, like the model itself, it depends on the quantum measurements that are made. Consequently all experimental data aiming at disproving Leggett's model should present evidence that the settings used in the experiment have been properly adjusted.

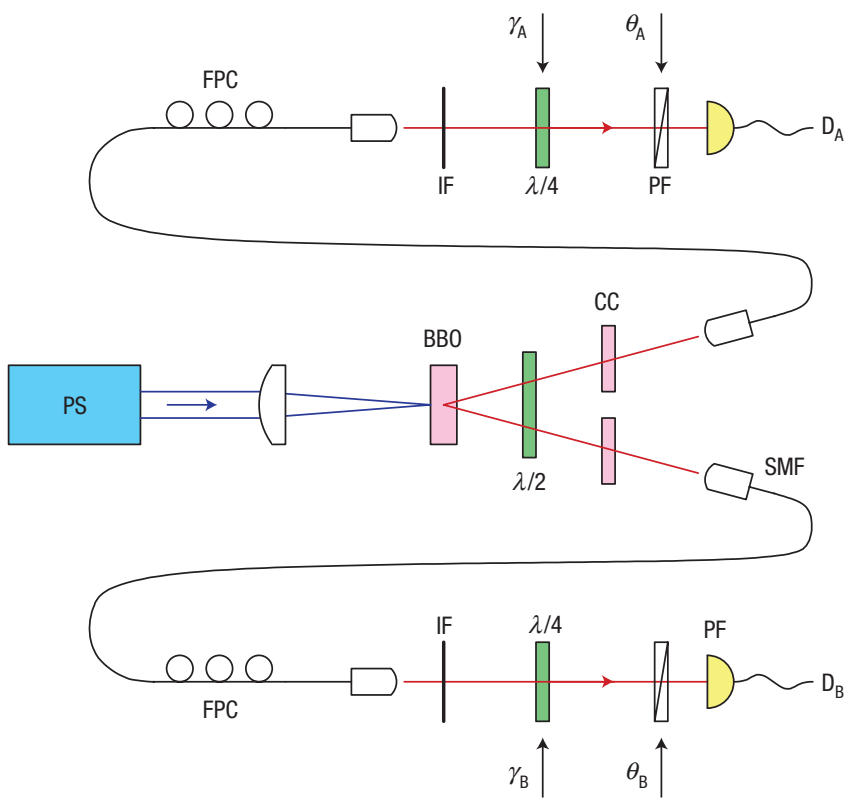

Figure 2 Experimental set-up. Polarization-entangled photon pairs are generated in a nonlinear optical crystal (BBO) and coupled into single-mode optical fibres (SMF), similarly as in ref. 9. Polarization measurements in arbitrary settings for each photon are made with polarization filters (PF) and quarter-wave plates $(\lambda / 4)$, followed by single-photon detectors $\left(D_{A, B}\right)$. More details can be found in Supplementary Information, section 1.

All previously available derivations of Leggett-type inequalities were quite lengthy and failed to suggest possible improvements or generalizations. We have found a much more straightforward derivation (see the Methods section), simply based on the fact that each $P_{\lambda}$ must be a valid probability distribution, so in particular $P_{\lambda}(\alpha, \beta \mid \mathbf{a}, \mathbf{b}) \geq 0$. Remarkably, this constraint of nonnegativity of probabilities, weak as it may seem, is enough to induce an observable incompatibility between Leggett's model and quantum predictions. In our derivation, it also seems that the previously derived Leggett-type inequalities are suboptimal; among the improved inequalities that our approach suggests, the simplest one reads

$$
\frac{1}{3} \sum_{i=1}^{3}\left|C\left(\mathbf{a}_{i}, \mathbf{b}_{i}\right)+C\left(\mathbf{a}_{i}, \mathbf{b}_{i}^{\prime}\right)\right| \equiv L_{3}(\varphi) \leq 2-\frac{2}{3}\left|\sin \frac{\varphi}{2}\right|,
$$

where $C(\mathbf{a}, \mathbf{b})=\sum_{\alpha, \beta} \alpha \beta P(\alpha, \beta \mid \mathbf{a}, \mathbf{b})$ is the usual correlation coefficient. This inequality holds provided the three measurements on Alice's side and six on Bob's fulfil some relations; a possible set of measurements is given in Fig. 1. For the singlet state, quantum mechanics predicts $C_{\Psi^{-}}(\mathbf{a}, \mathbf{b})=-\mathbf{a} \cdot \mathbf{b}$. Thus, for the settings defined in Fig. 1, $L_{3}(\varphi)$ is

$$
L_{\Psi^{-}}(\varphi)=2\left|\cos \frac{\varphi}{2}\right| .
$$

This expression violates inequality (4) for a large range of values $\varphi$.

To test the Leggett-type inequality (4) in an experiment, we prepared polarization-entangled photon pairs in single-mode optical fibres in a close approximation to a singlet state, similarly as in ref. 9. In our set-up (Fig. 2), we choose the settings of polarization measurements $\mathbf{a}_{i}, \mathbf{b}_{i}$ and $\mathbf{b}_{i}^{\prime}$ for the individual photons by dialling in appropriate orientation angles $\gamma_{\mathrm{A}, \mathrm{B}}$ for 


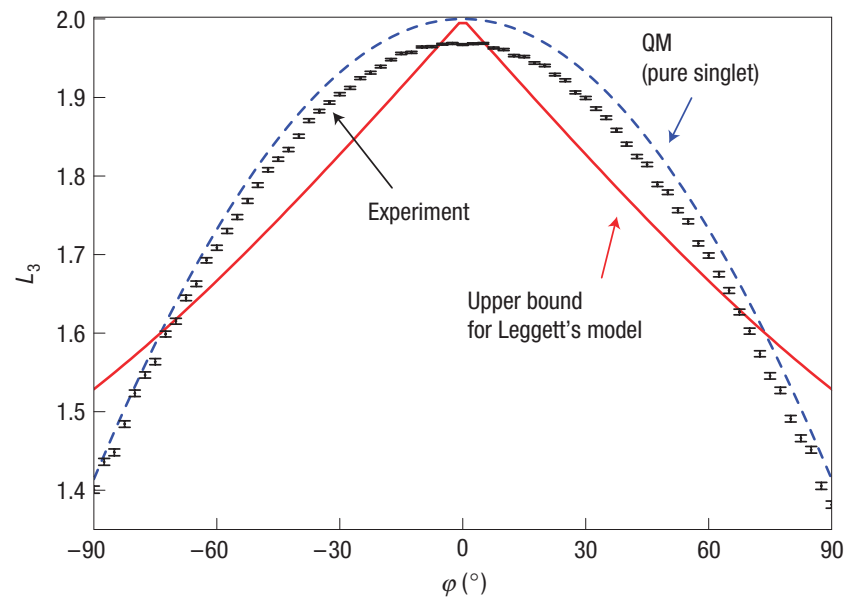

Figure 3 Violation of Leggett's model. The experimental values for $L_{3}$ over a range of separation angles $\varphi$ (points with error bars) violate the bound given by Leggett's model (solid line), and follow qualitatively the quantum-mechanical (QM) prediction (dashed line). The error bars indicate \pm 1 standard deviation of propagated Poissonian counting statistics assumed for photodetection events. The largest violations of inequality (4) are found for $\varphi= \pm 25^{\circ}$, with 40.6 and 38.1 standard deviations, respectively.

two quarter-wave plates $(\lambda / 4)$ and angles $\theta_{\mathrm{A}, \mathrm{B}}$ for two absorptive polarization filters. Details of the experimental implementation can be found in Supplementary Information, section 1. Through four consecutive coincidence measurements between photodetectors $\mathrm{D}_{\mathrm{A}, \mathrm{B}}$ for all combinations of settings $\mathbf{a},-\mathbf{a}$ and $\mathbf{b},-\mathbf{b}$, we establish an experimental value for a correlation coefficient $C(\mathbf{a}, \mathbf{b})$.

The correlation coefficients necessary to compose values for $L_{3}\left(\varphi= \pm 30^{\circ}\right)$ were obtained with an integration time of $T=60 \mathrm{~s}$ per point, leading to values of $1.9068 \pm 0.0009$ for $\varphi=-30^{\circ}$ and $1.9005 \pm 0.0010$ for $\varphi=30^{\circ}$. This corresponds to a violation of the bound for $L_{3}\left(\varphi=-30^{\circ}\right)$ and $L_{3}\left(\varphi=+30^{\circ}\right)$ in Leggett's model by 83.7 and 74.5 standard deviations, respectively.

The asymmetry in the measured values of $L_{3}(\varphi)$ is an indication for experimental imperfections in the accuracy of the settings, such as a possible misalignment of one of the quarter-wave plates with respect to the polarizing filters. To test this alignment, we collected values for $L_{3}$ over a larger range of $\varphi$ with an integration time of $T=15 \mathrm{~s}$ per setting (Fig. 3). The variation of $L_{3}$ with $\varphi$ is compatible with the quantum-mechanical prediction for a singlet state with residual coloured noise and an orientation uncertainty of the quarter-wave plate of $0.2^{\circ}$.

The falsification of Leggett's model proves that it is impossible to reconstruct quantum correlations from hypothetical, more elementary correlations in which individual properties would be sharply defined. Let us argue that a much stronger statement holds, namely, that individual properties cannot be even partially defined.

We first consider the following straightforward generalization of Leggett's model: we allow the 'local states' $\lambda$ to be mixed states, for example photons with a degree of polarization $\eta$. Therefore, we replace (2) and (3) by

$$
\begin{aligned}
& \langle\alpha\rangle_{\lambda}=\eta \mathbf{u} \cdot \mathbf{a}, \\
& \langle\beta\rangle_{\lambda}=\eta \mathbf{v} \cdot \mathbf{b},
\end{aligned} \quad \text { with } \quad 0<\eta \leq 1 .
$$

The derivation of inequalities for this model follows exactly the same pattern as for usual Leggett-type inequalities. In particular, the generalized version of (4) is

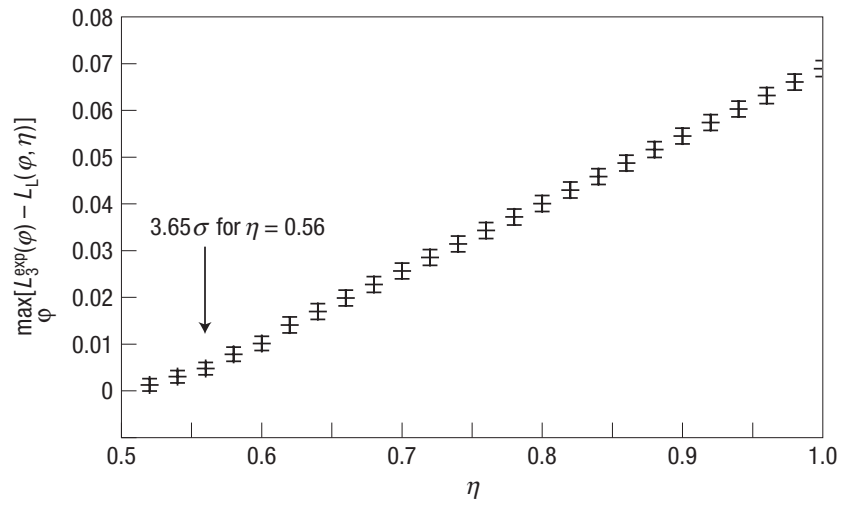

Figure 4 Experimental test of the generalized Leggett inequality for different degrees of purity $\eta$. From the measurement shown in Fig. 3, we extract the maximal excess of the experimental $L_{3}(\varphi)$ with respect to the upper bound (denoted $L_{L}$ ) in inequality (5), for various degrees of purity $\eta$. For $\eta<0.6$ data at $\varphi=-15^{\circ}$ are considered, whereas for $\eta>0.6$ the largest violations are observed for $\varphi=-25^{\circ}$.

$$
L_{3}(\varphi) \leq 2-\frac{2}{3} \eta\left|\sin \frac{\varphi}{2}\right|
$$

which, for angles $\varphi$ small enough, is violated by $L_{\Psi^{-}}(\varphi)$ for any value of $\eta>0$. Thus, as soon as the degree of purity of the 'local states' is non-zero, this generalization of Leggett's model also fails to reproduce quantum-mechanical predictions (see Supplementary Information, section 3 for a more complete analysis of this generalization).

Experimentally, we cannot expect to conduct a meaningful comparison between these two predictions down to $\eta=0$, owing to imperfections in the state preparation. From the measurement of Fig. 3, however, we can claim experimental evidence of a violation for all $\eta \geq 0.56$, with a statistical significance of at least 3.65 standard deviations, thus putting a lower bound for this class of models (Fig. 4).

It is then natural to conjecture that no model of form (1), with non-signalling correlations $P_{\lambda}$, can perfectly reproduce the correlations of the singlet state $C_{\Psi^{-}}(\mathbf{a}, \mathbf{b})=-\mathbf{a} \cdot \mathbf{b}$, unless

$$
\langle\alpha\rangle_{\lambda}=\langle\beta\rangle_{\lambda}=0
$$

for all measurements $\mathbf{a}$ and $\mathbf{b}$ (except perhaps for a few $\lambda$ in a zero-measure set). In the Methods section we prove this conjecture for models with discrete supplementary variables $\lambda$; continuity arguments should enable us to extend the result to more general models. We thus have a necessary condition for a non-signalling model to be compatible with quantum mechanics. However, given a model with non-trivial marginals, finding an explicit inequality that can be tested experimentally against quantum predictions is another problem; for this, we need the specific details of the model.

In summary, with the general goal of improving our understanding of quantum correlations, we reformulated Leggett's model. No time ordering of the events was assumed, and all assumptions were made on the local part of the correlations. We derived new Leggett-type inequalities, simpler and stronger than previously known. The simplest version of our inequalities has been experimentally violated. Finally we investigated more general models à la Leggett, for which we imposed only the no-signalling condition. We argued that any such model with biased marginals is 
incompatible with quantum predictions for the singlet state. This shows that quantum correlations cannot be reconstructed from 'simpler' correlations in which the individual properties would be even partially defined. Nature is really such that, in some cases, individual properties are completely lost whereas global properties are sharply defined.

Our result is in good agreement with the recent work in of Colbeck and Renner ${ }^{14}$, who have derived general inequalities to falsify such models with non-trivial marginals. An example of a non-signalling model that successfully reproduces the singlet correlations can be found in ref. 15; indeed, this model has unbiased marginals. It is also worth mentioning the non-local model of Toner and Bacon ${ }^{16}$, which reproduces analytically the singlet correlations with one bit of communication. In this model, the probability distributions $P_{\lambda}$ have non-vanishing marginals; however, the $P_{\lambda}$ are signalling. The remarkable property of the Toner-Bacon model is that the communication is cleverly hidden, such that the final probability distribution $P$ is non-signalling.

This work is part of the general research programme that looks for non-local models compatible or incompatible with quantum predictions. The goal is to find out what is essential in quantum correlations. Here we have found that to simulate or to decompose the singlet correlations we cannot use non-signalling correlations $P_{\lambda}$ with non-trivial marginals. This nicely complements the result of ref. 17, that the correlations corresponding to very partially entangled states, hence large marginals, cannot be simulated by a single PR box, which has trivial marginals.

\section{METHODS}

\section{SIMPLE DERIVATION OF INEQUALITIES THAT TEST LEGGETT'S MODEL}

Convenient notations. As mentioned, in this paper we focus on the case of binary outcomes $\alpha, \beta= \pm 1$. In this case, the correlations can conveniently be written as

$$
P_{\lambda}(\alpha, \beta \mid \mathbf{a}, \mathbf{b})=\frac{1}{4}\left(1+\alpha M_{\lambda}^{\mathrm{A}}(\mathbf{a}, \mathbf{b})+\beta M_{\lambda}^{\mathrm{B}}(\mathbf{a}, \mathbf{b})+\alpha \beta C_{\lambda}(\mathbf{a}, \mathbf{b})\right) .
$$

This expression enables us to clearly distinguish the marginals $M_{\lambda}^{\mathrm{A}}(\mathbf{a}, \mathbf{b})=\sum_{\alpha, \beta} \alpha P_{\lambda}(\alpha, \beta \mid \mathbf{a}, \mathbf{b})$ on Alice's side and $M_{\lambda}^{\mathrm{B}}(\mathbf{a}, \mathbf{b})=\sum_{\alpha \beta} \beta P_{\lambda}(\alpha, \beta \mid \mathbf{a}, \mathbf{b})$ on Bob's, and the correlation coefficient $C_{\lambda}(\mathbf{a}, \mathbf{b})=\sum_{\alpha, \beta} \alpha \beta P_{\lambda}(\alpha, \beta \mid \mathbf{a}, \mathbf{b})$. Throughout the Methods section, we shall use these notations; in the main text, we have used more standard and simplified notations, the correspondence being $\langle\alpha\rangle_{\lambda}=M_{\lambda}^{\mathrm{A}}(\mathbf{a}, \mathbf{b})$, $\langle\beta\rangle_{\lambda}=M_{\lambda}^{\mathrm{B}}(\mathbf{a}, \mathbf{b})$. The no-signalling condition is $M_{\lambda}^{\mathrm{A}}(\mathbf{a}, \mathbf{b})=M_{\lambda}^{\mathrm{A}}(\mathbf{a})$ and $M_{\lambda}^{\mathrm{B}}(\mathbf{a}, \mathbf{b})=M_{\lambda}^{\mathrm{B}}(\mathbf{b})$.

In order for the decomposition (1) to be a valid mixture of correlations, all distributions $P_{\lambda}$ should be non-negative. As we said, this constraint is enough to derive Leggett-type inequalities. From equation (6), we can see that the non-negativity implies the general constraints

$$
\left|M_{\lambda}^{\mathrm{A}}(\mathbf{a}) \pm M_{\lambda}^{\mathrm{B}}(\mathbf{b})\right| \leq 1 \pm C_{\lambda}(\mathbf{a}, \mathbf{b})
$$

Constraints on the marginals $M_{\lambda}^{\mathrm{A}}$ or $M_{\lambda}^{\mathrm{B}}$ thus imply constraints on the correlation coefficients $C_{\lambda}$, and vice versa.

Let us now consider one measurement setting a for Alice and two measurement settings $\mathbf{b}, \mathbf{b}^{\prime}$ for Bob, and let us combine the previous inequalities (7) that we get for $(\mathbf{a}, \mathbf{b})$ and $\left(\mathbf{a}, \mathbf{b}^{\prime}\right)$. Using the triangle inequality, we get

$$
\left|C_{\lambda}(\mathbf{a}, \mathbf{b}) \pm C_{\lambda}\left(\mathbf{a}, \mathbf{b}^{\prime}\right)\right| \leq 2-\left|M_{\lambda}^{\mathrm{B}}(\mathbf{b}) \mp M_{\lambda}^{\mathrm{B}}\left(\mathbf{b}^{\prime}\right)\right| .
$$

These constraints must hold for all probability distributions $P_{\lambda}$. After integration over the $\lambda$, we get, for the averaged correlation coefficients $C(\mathbf{a}, \mathbf{b})=\int \mathrm{d} \lambda \rho(\lambda) C_{\lambda}(\mathbf{a}, \mathbf{b})$,

$$
\left|C(\mathbf{a}, \mathbf{b}) \pm C\left(\mathbf{a}, \mathbf{b}^{\prime}\right)\right| \leq 2-\int \mathrm{d} \lambda \rho(\lambda)\left|M_{\lambda}^{\mathrm{B}}(\mathbf{b}) \mp M_{\lambda}^{\mathrm{B}}\left(\mathbf{b}^{\prime}\right)\right| .
$$

This inequality is general for all models with 'local marginals', that is, that fulfil the no-signalling condition.

Derivation of a simple Leggett-type inequality. Now we derive an inequality satisfied by Leggett's specific model, that can be experimentally tested. Inequality (8) implies, for the particular form of equation (3) for Bob's marginals,

$$
\left|C(\mathbf{a}, \mathbf{b})+C\left(\mathbf{a}, \mathbf{b}^{\prime}\right)\right| \leq 2-\int \mathrm{d} \lambda \rho(\lambda)\left|\mathbf{v} \cdot\left(\mathbf{b}-\mathbf{b}^{\prime}\right)\right| .
$$

Let us consider three triplets of settings $\left(\mathbf{a}_{i}, \mathbf{b}_{i}, \mathbf{b}_{i}^{\prime}\right)$, with the same angle $\varphi$ between all pairs $\left(\mathbf{b}_{i}, \mathbf{b}_{i}^{\prime}\right)$, and such that $\mathbf{b}_{i}-\mathbf{b}_{i}^{\prime}=2 \sin (\varphi / 2) \mathbf{e}_{i}$, where $\left\{\mathbf{e}_{1}, \mathbf{e}_{2}, \mathbf{e}_{3}\right\}$ form an orthogonal basis (Fig. 1). After combining the three corresponding inequalities (9), using the fact that $\sum_{i=1}^{3}\left|\mathbf{v} \cdot \mathbf{e}_{i}\right| \geq 1$ and the normalization $\int \mathrm{d} \lambda \rho(\lambda)=1$, we finally get the Leggett-type inequality (4).

For a pure singlet state, inequality (4) is violated when $|\varphi|<4 \arctan (1 / 3) \simeq 73.7^{\circ}$, and the maximal violation is obtained for $|\varphi|=2 \arctan (1 / 3) \simeq 36.9^{\circ}$. In the case of imperfect interference visibility $V\left(\tilde{L}_{\Psi^{-}}(\varphi)=2 V|\cos (\varphi / 2)|\right)$, a violation can still be observed as long as $V>V_{\mathrm{th}}^{(3)}=\sqrt{1-(1 / 3)^{2}}=(2 \sqrt{2} / 3) \simeq 94.3 \%$.

Note that other Leggett-type inequalities can be derived, as we mention in Supplementary Information, section 2.

\section{ANY NON-SIGNALLING MODEL MUST HAVE VANISHING MARGINALS}

We prove here that all marginals in a non-signalling model must necessarily satisfy the constraints (11) and (12) below; and we argue that this in turn implies the claim made in the main text, namely, all the marginals must vanish (apart perhaps on a zero-measure subset of $\lambda$ ).

In order for a general non-signalling model to reproduce all quantum correlations $C_{\Psi^{-}}(\mathbf{a}, \mathbf{b})=-\mathbf{a} \cdot \mathbf{b}$ of the singlet state, we must have, according to equation (8), for all $\mathbf{a}, \mathbf{b}, \mathbf{b}^{\prime}$ on the Poincaré sphere

$$
\int \mathrm{d} \lambda \rho(\lambda)\left|M_{\lambda}^{\mathrm{B}}(\mathbf{b}) \pm M_{\lambda}^{\mathrm{B}}\left(\mathbf{b}^{\prime}\right)\right| \leq 2-\left|\mathbf{a} \cdot\left(\mathbf{b} \mp \mathbf{b}^{\prime}\right)\right|
$$

and therefore, for all $\mathbf{b}, \mathbf{b}^{\prime}$,

$$
\int \mathrm{d} \lambda \rho(\lambda)\left|M_{\lambda}^{\mathrm{B}}(\mathbf{b}) \pm M_{\lambda}^{\mathrm{B}}\left(\mathbf{b}^{\prime}\right)\right| \leq 2-\left\|\mathbf{b} \mp \mathbf{b}^{\prime}\right\|,
$$

where $\|\cdot\|$ is the Euclidian norm.

In the case where $\mathbf{b}^{\prime}=-\mathbf{b}$, the first constraint of equation (10) implies that for all $b$

$$
\int \mathrm{d} \lambda \rho(\lambda)\left|M_{\lambda}^{\mathrm{B}}(\mathbf{b})+M_{\lambda}^{\mathrm{B}}(-\mathbf{b})\right|=0 .
$$

In the case $\mathbf{b}^{\prime} \rightarrow \mathbf{b}$, the two vectors being normalized, we have $2-\left\|\mathbf{b}+\mathbf{b}^{\prime}\right\|=2-\sqrt{4-\left\|\mathbf{b}-\mathbf{b}^{\prime}\right\|^{2}} \sim\left(\left\|\mathbf{b}-\mathbf{b}^{\prime}\right\|^{2} / 4\right)$ and therefore the second constraint of (10) implies that for all $\mathbf{b}$

$$
\lim _{\mathbf{b}^{\prime} \rightarrow \mathbf{b}} \int \mathrm{d} \lambda \rho(\lambda) \frac{\left|M_{\lambda}^{\mathrm{B}}(\mathbf{b})-M_{\lambda}^{\mathrm{B}}\left(\mathbf{b}^{\prime}\right)\right|}{\left\|\mathbf{b}-\mathbf{b}^{\prime}\right\|}=0 .
$$

Let us now prove, in the case of discrete $\lambda\left(\lambda \in\left\{\lambda_{i}\right\}\right)$, that (11) and (12) in turn necessarily imply that the marginals $M_{\lambda}^{\mathrm{B}}(\mathbf{b})$ must vanish. In this case, the integral $\int \mathrm{d} \lambda \rho(\lambda)$ should be changed to a discrete sum $\sum_{i} p_{\lambda_{i}}$.

Constraint (11) indeed implies that for all $\lambda_{i}$ (such that $p_{\lambda_{i}}>0$ ) and for all $\mathbf{b}, M_{\lambda_{i}}^{\mathrm{B}}(-\mathbf{b})=-M_{\lambda i}^{\mathrm{B}}(\mathbf{b})$, that is, $M_{\lambda i}^{\mathrm{B}}$ must be an odd function of $\mathbf{b}$. This is a very natural property that we would impose on such a model; in particular, Leggett's model indeed has odd marginals.

In addition, constraint (12) implies that for all $\lambda_{i}$ and for all $\mathbf{b}$, $\lim _{\mathbf{b}^{\prime} \rightarrow \mathbf{b}}\left(\left|M_{\lambda_{i}}^{\mathrm{B}}(\mathbf{b})-M_{\lambda_{i}}^{\mathrm{B}}\left(\mathbf{b}^{\prime}\right)\right| /|| \mathbf{b}-\mathbf{b}^{\prime}||\right)=0$, that is, that all $M_{\lambda_{i}}^{\mathrm{B}}$ are differentiable, and their derivative is zero for all $\mathbf{b}$ : therefore, they are constant. As they have to be odd functions, then necessarily they are equal to zero.

In conclusion, for discrete $\lambda$, Bob's marginals $M_{\lambda_{i}}^{\mathrm{B}}(\mathbf{b})$ must all vanish; of course, the same reasoning holds for Alice's marginals $M_{\lambda_{i}}^{\mathrm{A}}(\mathbf{a})$. This result should also be valid for any distribution $\rho(\lambda)$, at least those physically reasonable (for example piecewise continuous): we conjecture that for any reasonable model to reproduce the quantum correlations of the singlet state, 
the marginals must necessarily vanish, in the sense that

$$
\begin{aligned}
& \forall \mathbf{a}, \quad \int \mathrm{d} \lambda \rho(\lambda)\left|M_{\lambda}^{\mathrm{A}}(\mathbf{a})\right|=0, \\
& \forall \mathbf{b}, \quad \int \mathrm{d} \lambda \rho(\lambda)\left|M_{\lambda}^{\mathrm{B}}(\mathbf{b})\right|=0,
\end{aligned}
$$

that is, for all $\mathbf{a}, \mathbf{b},\left|M_{\lambda}^{\mathrm{A}}(\mathbf{a})\right|=\left|M_{\lambda}^{\mathrm{B}}(\mathbf{b})\right|=0$ for 'almost all' $\lambda$ (except for a zero-measure subset of the $\lambda$, that could possibly depend on $\mathbf{a}, \mathbf{b})$.

Received 30 January 2008; accepted 6 June 2008; published 6 July 2008.

\section{References}

1. Bell, J. S. On the Einstein-Podolsky-Rosen Paradox. Physics 1, 195-200 (1964).

2. Bell, J. S. Speakable and Unspeakable in Quantum Mechanics 152 (Cambridge Univ. Press, Cambridge, 1987).

3. Barrett, J., Hardy, L. \& Kent, A. No signaling and quantum key distribution. Phys. Rev. Lett. 95, $010503(2005)$.

4. Acín, A., Gisin, N. \& Masanes, L. From Bell's theorem to secure quantum key distribution. Phys. Rev Lett. 97, 120405 (2006)

5. Acín, A. et al. Device-independent security of quantum cryptography against collective attacks. Phys. Rev. Lett. 98, 230501 (2007).

6. Leggett, A. J. Nonlocal hidden-variable theories and quantum mechanics: An incompatibility theorem. Found. Phys. 33, 1469-1493 (2003).

7. Gröblacher, S. et al. An experimental test of non-local realism. Nature 446, 871-875 (2007).

8. Paterek, T. et al. Experimental test of nonlocal realistic theories without the rotational symmetry assumption. Phys. Rev. Lett. 99, 210406 (2007).
9. Branciard, C. et al. Experimental falsification of Leggett's nonlocal variable model. Phys. Rev. Lett. 99, 210407 (2007).

10. Popescu, S. \& Rohrlich, D. Quantum nonlocality as an axiom. Found. Phys. 24, 379-385 (1994).

11. Stefanov, A., Zbinden, H., Gisin, N. \& Suarez, A. Quantum correlations with spacelike separated beam splitters in motion: Experimental test of multisimultaneity. Phys. Rev. Lett. 88, 120404 (2002). 12. Stefanov, A., Zbinden, H., Gisin, N. \& Suarez, A. Quantum entanglement with acousto-optic modulators: Two-photon beats and Bell experiments with moving beam splitters. Phys. Rev. A 67, 042115 (2003).

13. Suarez, A. Nonlocal 'realistic' Leggett models can be considered refuted by the before-before experiment. Found. Phys. 38, 583-589 (2008).

14. Colbeck, R. \& Renner, R. Hidden variable models for quantum theory cannot have any local part. Preprint at $<$ http://arxiv.org/abs/0801.2218> (2008).

15. Cerf, N. J., Gisin, N., Massar, S. \& Popescu, S. Simulating maximal quantum entanglement without communication. Phys. Rev. Lett. 94, 220403 (2005).

16. Toner, B. F. \& Bacon, D. Communication cost of simulating Bell correlations. Phys. Rev. Lett. 91, 187904 (2003).

17. Brunner, N., Gisin, N. \& Scarani, V. Entanglement and non-locality are different resources. New J. Phys. 7, 88 (2005).

Supplementary Information accompanies this paper on www.nature.com/naturephysics.

\section{Acknowledgements}

We thank A. Ekert and C. Simon for comments. C.B., N.B. and N.G. acknowledge financial support from the EU project QAP (IST-FET FP6-015848) and Swiss NCCR Quantum Photonics. A.L.L. and C.K. acknowledge ASTAR for financial support under SERC grant No 0521010043 . This work is partly supported by the National Research Foundation and Ministry of Education, Singapore.

Author information

Reprints and permission information is available online at http://npg.nature.com/reprintsandpermissions. Correspondence and requests for materials should be addressed to C.B. 